\title{
Parametric Study on Vibration and Harmonic Analysis of Moderately Thick Functionally Graded Plates Using FEM
}

\author{
Avadesh K. Sharma ${ }^{1, a}$, M K Gaur ${ }^{2}$ and R K Dwivedi ${ }^{3}$ \\ ${ }^{1}$ Associate Professor, Rajkiya Engineering College, Mainpuri, 205119 \\ ${ }^{2}$ Professor, Madhav Institute of Technology \& Science, Gwalior \\ ${ }^{3}$ Associate Professor, Maulana Azad National Institute of Technology, Bhopal
}

\begin{abstract}
Finite element method is used to investigate the free vibration and harmonic analysis of functionally graded plates. The material properties of the plates are assumed to vary continuously through their thickness direction according to a power-law distribution of the volume fractions of the plate constituents. The four noded shell 181 elements are used to analyse the functionally graded plates. The aim is to fill the void in the available literature with respect to the free vibration results of Functionally Graded plates. Convergence and Comparison studies with respect to the number of nodes has been carried out using FEM. The natural frequency, mode shape and harmonic analysis of FG plate has been determined using finite element package ANSYS.
\end{abstract}

\section{Introduction}

Composite materials have been widely used in aircraft and other engineering applications for many years because of their excellent strength-to-weight and stiffness-to-weight ratios. Functionally graded materials (FGM) are a class of composite materials that were first proposed by Bever and Duwez [1] in 1972. In a typical FGM plate the material properties continuously vary over the thickness direction by mixing two different materials [2], usually ceramic and metal. The gradual variation of properties avoids the delamination failure that are common in laminated composites. The computational modelling of FGM is an important tool to the understanding of the structures behavior, and has been the target of intense research, from micro to macro mechanics [3-6]. Researchers have also turned their attention to the vibration and dynamic response of functionally graded structures [7-9]. Sheng and Wang [10] investigated the effect of thermal load on vibration, buckling and dynamic stability of functionally graded cylindrical shells embedded in an elastic medium. A review of the main developments in FGM can be found in Birman and Byrd [11]. Sharma et. al. [12] presented the free vibration of shear-deformable antisymmetric angle-ply laminated rectangular plates having translational as well as rotational edge constraints. The Vibration attenuation using functionally graded material is studied by Saeed et. al. [13].

The vibration and harmonic responses of composite plates and FGMs have been extensively studied by a number of researchers [14-16]. However, the aim of the work is to study the effect of functionally graded materials on the vibration behaviour by doing modal and harmonic analysis of the models.

\footnotetext{
${ }^{\text {a }}$ Corresponding author : sharma.avadesh@gmail.com
} 


\section{Functionally Graded Material Properties}

A functionally graded material plate as shown in fig. 1 is considered to be a plate of uniform thickness that is made of ceramic and metal. The material property is assumed to be graded through the thickness according to a Power-Law distribution that is

$$
P(z)=\left(P_{c}-P_{m}\right) V_{m}+P_{m}
$$

where $P m, P c, V m$ and $V c$ are the material properties and the volume fraction of the metal and ceramic, respectively, the compositions represent in relation to

$$
V_{c}+V_{m}=1
$$

The volume fraction of ceramic $\left(V_{C}\right)$ can then be written as follows:

$$
V_{C}=\left(\frac{z}{h}+\frac{1}{2}\right)^{n} \quad(n \geq 0)
$$

where the positive number $n(0 \leq n \leq \infty)$ is the power law or the volume fraction index. $z$ is a distance parameter along the graded direction, while, $h$ is the total length of the direction. To find out the results of material properties according to the power law distribution, this can be achieved by substituting the equations of material volume fractions Eq. (2) and Eq. (3) into Eq. (1).
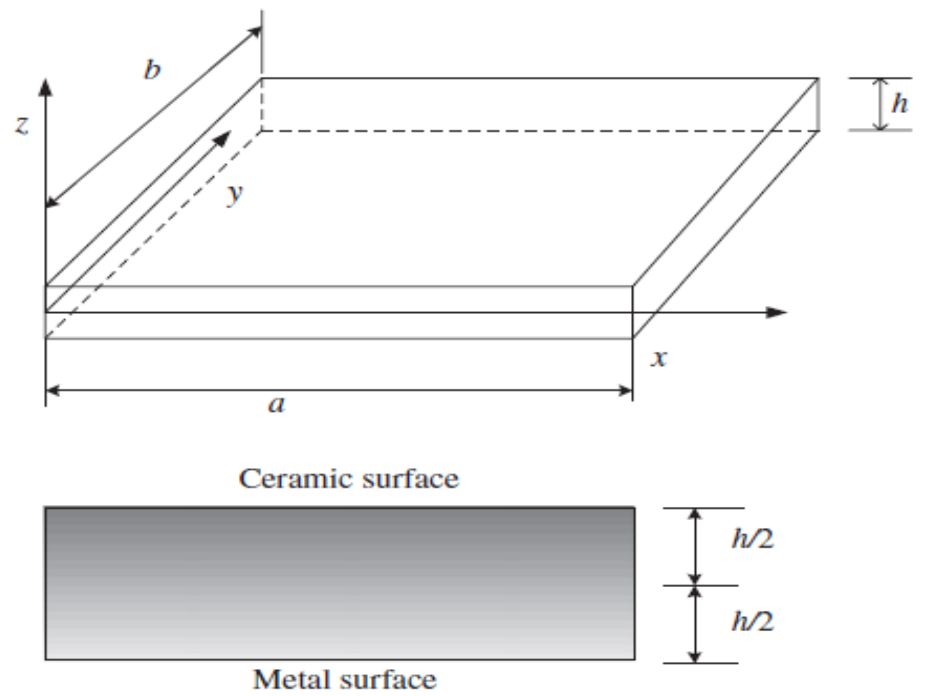

Figure 1. Geometry of Functionally graded plate

\section{Results and Discussion}

\subsection{Modal Analysis}

The variation of the frequency parameter with the boundary condition for functionally graded plates is described in Tables 1-2 respectively. It shows a comparison of the fundamental natural frequency parameters of functionally graded plates for volume fraction exponent $n=0$. It can be seen that the as the mesh size increases the results are converging and convergence are achived at the mesh size of 19x19. The results are compared with the Zhao et. Al. [17]. 
Table 1: Variation of the frequency parameter with the volume fraction exponent $n=0$ for square FG plates $(\mathrm{a} / \mathrm{h}=10)$ with $\mathrm{CFFF}$

\begin{tabular}{|l|l|l|l|l|l|l|l|l|l|l|}
\hline $3 \times 3$ & 11.7174 & 22.4627 & 26.6912 & 33.8398 & 37.9197 & 38.1226 & 42.2459 & 45.4010 & 45.7544 & 51.0061 \\
\hline $5 \times 5$ & 10.5809 & 20.7963 & 20.9147 & 29.6169 & 35.8369 & 36.0877 & 37.7263 & 37.7364 & 43.2174 & 44.1762 \\
\hline $7 \times 7$ & 10.3100 & 19.9219 & 19.9483 & 28.2032 & 33.6206 & 33.8827 & 37.6205 & 37.6217 & 40.7289 & 40.8631 \\
\hline $9 \times 9$ & 10.2299 & 19.7064 & 19.7360 & 27.8315 & 33.0467 & 33.3661 & 37.5852 & 37.5877 & 40.0541 & 40.1108 \\
\hline $11 \times 11$ & 10.1758 & 19.5785 & 19.5823 & 27.6009 & 32.7228 & 33.0107 & 37.5606 & 37.5625 & 39.6554 & 39.6894 \\
\hline $13 \times 13$ & 10.1512 & 19.5149 & 19.5256 & 27.4901 & 32.5559 & 32.8432 & 37.5486 & 37.5499 & 39.4632 & 39.4701 \\
\hline $15 \times 15$ & 10.0340 & 19.2276 & 19.2295 & 27.0157 & 31.8887 & 32.2346 & 37.5341 & 37.5348 & 38.6379 & 38.6492 \\
\hline $17 \times 17$ & 10.0006 & 19.1325 & 19.1369 & 26.8727 & 31.6978 & 32.0229 & 37.5260 & 37.5266 & 38.3941 & 38.3985 \\
\hline $19 \times 19$ & 9.9918 & 19.1098 & 19.1111 & 26.8317 & 31.6556 & 31.9637 & 37.5215 & 37.5222 & 38.3361 & 38.3412 \\
\hline $21 \times 21$ & 9.9830 & 19.0859 & 19.0865 & 26.7914 & 31.6021 & 31.9101 & 37.5171 & 37.5171 & 38.2631 & 38.2649 \\
\hline $23 \times 23$ & 9.9666 & 19.0487 & 19.0518 & 26.7429 & 31.5258 & 31.8408 & 37.5089 & 37.5096 & 38.1824 & 38.1887 \\
\hline $\begin{array}{l}\text { Zhao et. } \\
\text { al [16] }\end{array}$ & 9.6329 & 18.313 & 18.313 & 25.499 & & & & & & \\
\hline
\end{tabular}

Boundary conditions at different mesh size:

Table 2: Variation of the frequency parameter with the volume fraction exponent $\mathrm{n}=0$ for square FG plates $(\mathrm{a} / \mathrm{h}=10)$ with

\begin{tabular}{|c|c|c|c|c|c|c|c|c|c|c|}
\hline $3 \times 3$ & 1.077363 & 2.617083 & 6.68115 & 6.6994 & 8.2656 & 9.61884 & 15.98373 & 16.70256 & 17.88 & 19.90044 \\
\hline $5 \times 5$ & 1.059282 & 2.504943 & 6.292314 & 6.6389 & 7.95501 & 8.95734 & 15.30396 & 15.91632 & 17.56 & 17.79309 \\
\hline $7 \times 7$ & 1.054494 & 2.489193 & 6.228243 & 6.6289 & 7.86429 & 8.80803 & 14.93163 & 15.90057 & 17.05 & 17.77923 \\
\hline $9 \times 9$ & 1.0521 & 2.481381 & 6.200838 & 6.6238 & 7.83846 & 8.75952 & 14.81823 & 15.89175 & 16.91 & 17.68851 \\
\hline $11 \times 11$ & 1.050777 & 2.476467 & 6.18786 & 6.6213 & 7.82019 & 8.73243 & 14.75334 & 15.88671 & 16.84 & 17.61984 \\
\hline $13 \times 13$ & 1.049706 & 2.472498 & 6.17778 & 6.62 & 7.81074 & 8.71101 & 14.70483 & 15.88419 & 16.8 & 17.58204 \\
\hline $15 \times 15$ & 1.046745 & 2.459205 & 6.142626 & 6.6182 & 7.77357 & 8.64045 & 14.55552 & 15.88104 & 16.62 & 17.43336 \\
\hline $17 \times 17$ & 1.046052 & 2.457819 & 6.133995 & 6.6175 & 7.76412 & 8.62974 & 14.53158 & 15.87915 & 16.58 & 17.40375 \\
\hline $19 \times 19$ & 1.045737 & 2.456937 & 6.131727 & 6.6169 & 7.76223 & 8.62533 & 14.52213 & 15.87789 & 16.57 & 17.39241 \\
\hline $\begin{array}{l}\text { Zhao et. } \\
\text { al [16 ] }\end{array}$ & 1.0298 & 2.3907 & 6.0047 & 7.6356 & & & & & & \\
\hline
\end{tabular}

CCCC Boundary conditions at different mesh size:

Table 3: frequency response amplitude of FG square plate with different volume fraction exponent

\begin{tabular}{|l|l|l|c|c|}
\hline B.C. & $\begin{array}{l}\text { Volume } \\
\text { fraction } \\
\text { exponent }\end{array}$ & Mode & Frequency (Hz) & Amplitude (m) \\
\hline CCCC & $n=0$ & Mode 1 & 1592.7 & 0.0000000146726 \\
\hline CCCC & $n=1$ & Mode 1 & 1324.5 & 0.0000000247805 \\
\hline CCCC & $n=5$ & Mode 1 & 858.76 & 0.0000000699683 \\
\hline CCCC & $n=10$ & Mode 1 & 811.53 & 0.0000000793071 \\
\hline
\end{tabular}

\subsection{Harmonic Analysis}

To know FRF plot, harmonic analysis is to be done by providing the range of natural frequency of 0 $\mathrm{Hz}$ to $2000 \mathrm{~Hz}$ and 100 substeps. It will generate FRF plot (linear) on graph of amplitude to frequency, 
frequency $(\mathrm{Hz})$ is taken on the $\mathrm{x}$-axis and amplitude $(\mathrm{m})$ on the $\mathrm{y}$-axis. From this graph we come to know its resonance point. Also many details of system such as amount of displacement, by how much frequency by how much amount system excited. It can be said that the overall response of system can be known. It is clear from the Table 1 that as the volume fraction exponent increases the frequency response amplitude of FG plates increases.

These frequency response amplitudes can also be reduced by providing damping constant. In the study, constant damping ratio of 0.01 is taken. Effect of volume fraction exponent on the frequency response amplitude for different sets of FG plates is shown in Figure 2-5 respectively. It is clear from the figures that as the volume fraction exponent increases the frequency response amplitudes increases. Figure 6 shows the first four modes of functionality graded plates.

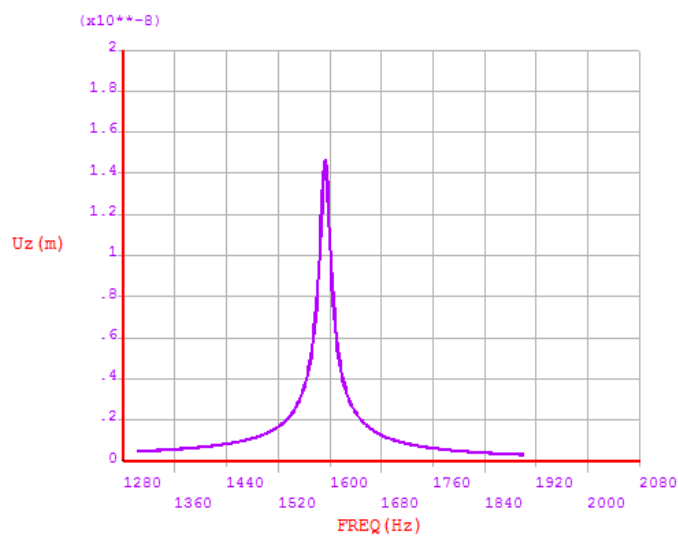

Figure 2. shows the FRF plots without damping FG plate at $\mathrm{CCCC}$ boundary condition at $\mathrm{n}=0$.

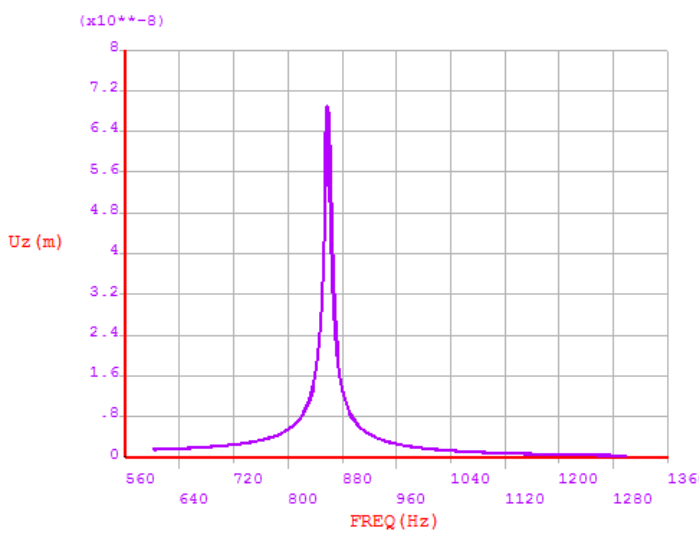

Figure 4. shows the FRF plots without damping FG plate at CCCC boundary condition at $n=5$.

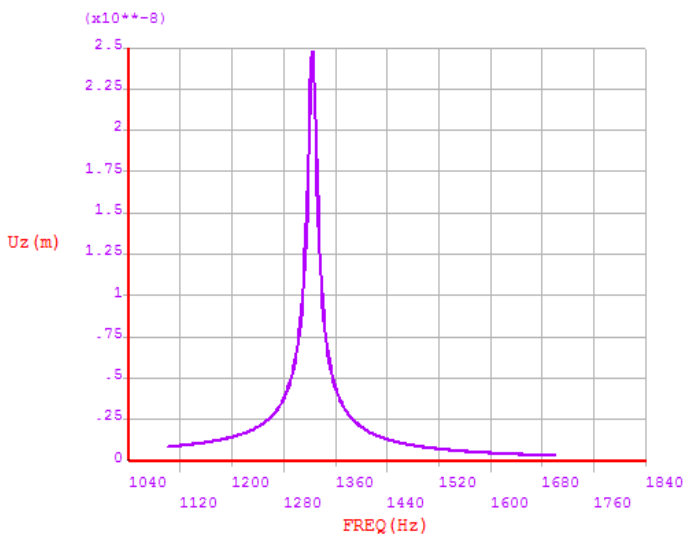

Figure 3. shows the FRF plots without damping FG plate at CCCC boundary condition at $n=1$.

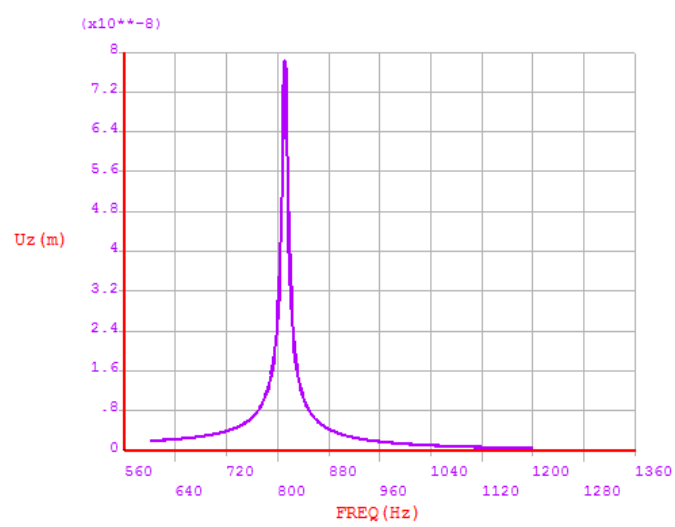

Figure 5. shows the FRF plots without damping FG plate at $\mathrm{CCCC}$ boundary condition at $\mathrm{n}=10$. 

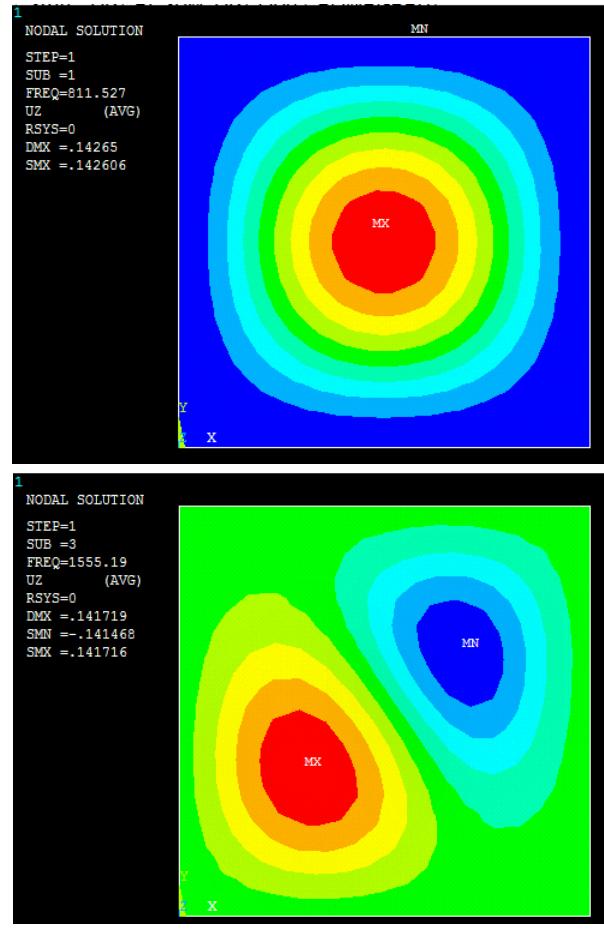

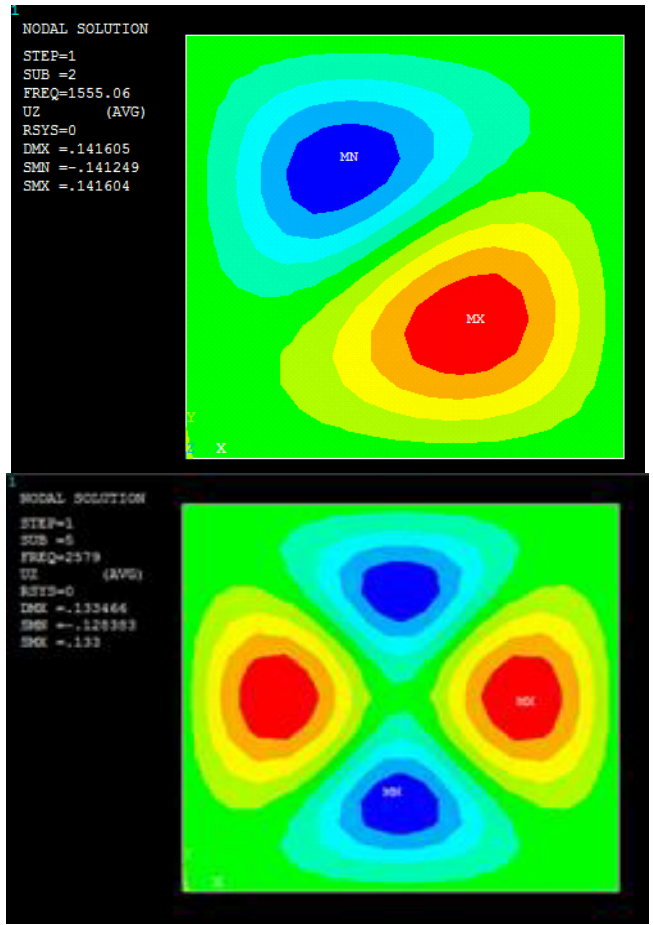

Figure 6. First four natural frequencies of functionally graded plates at volume fraction exponent $\mathrm{n}=10$

\section{Conclusion}

In this study, vibration and harmonic analysis of FGM plates are analysed. Four functionally graded plates of different sets of materials are considered and their natural frequencies and frequency response amplitude at the fundamental mode are determined. Convergence tests and comparison studies have been carried out with the commercially available software (ANSYS). A four noded layered shell element (SHELL181) is used throughout the problem. The obtained results have illustrated a good agreement with those available in the literature for different volume fraction indices. It is concluded that as the volume fraction exponent increases the frequency response amplitude of FG plates increases and results also shows that for all the Functionally graded plates the frequencies decreases as the volume fraction exponent increases.

\section{References}

1. M.B. Bever and P.E. Duwez. Gradients in composite materials. Materials Science and Engineering, 10(0):1 - 8, 1972.

2. Y. Miyamoto, W.A. Kaysser, B.H. Rabin, A. Kawasaki, and R.G. Ford. Functionally Graded Materials: Design, Processing and Applications. Kluwer Academic Publishers, 1999.

3. F.J. Ferrante and L.L. Graham-Brady. Stochastic simulation of non-gaussian/non-stationary properties in a functionally graded plate. Computer Methods in Applied Mechanics and Engineering, 194(12-16):1675 - 1692, 2005.

4. H.M Yin, L.Z Sun, and G.H Paulino. Micromechanics-based elastic model for functionally graded materials with particle interactions. Acta Materialia, 52(12):3535 - 3543, 2004.

5. Zheng Zhong and Ertao Shang. Closed-form solutions of three-dimensional functionally graded plates. Mechanics of Advanced Materials and Structures, 15(5):355-363, 2008. 
6. T. K. Nguyen, K. Sab, and G. Bonnet. Shear correction factors for functionally graded plates. Mechanics of Advanced Materials and Structures, 14(8):567-575, 2007.

7. H.L.Dai,X.Wang,Thermo-electro-elastic transient responses in piezoelectric hollow structures, International Journal of Solids and Structures 42 (2005) 1151-1171.

8. X.L.Huang, .H.Shen, Nonlinear vibration and dynamic response of functionally graded plates in thermal environments, International Journal of Solids and Structures 41 (2004)2403-2427.

9. J.Yang,S.H. Shen, Free vibration and parametric resonance of shear deformable functionally graded cylindrical panels, Journal of Sound and Vibration 261 (2003) 871-893.

10. G.G.Sheng, X. Wang, Thermal vibration, buckling and dynamic stability of functionally graded cylindrical shells embedded in an elastic medium, Journal of Reinforced Plastics and Composites 27 (2008)117-134.

11. Victor Birman and Larry W. Byrd. Modeling and analysis of functionally graded materials and structures. Applied Mechanics Reviews, 60(5):195-216, 2007.

12. Sharma A. K. \& Mittal N.D., Free vibration analysis of moderately thick Anti-symmetric angleply laminated rectangular plates with elastic edge constraints, Mechanics of Advanced Materials and Structures, 21, 341-348, 2014.

13. Asiri Saeed, Hedia Hassan \& EissaWael, Vibration attenuation using functionally graded material. World Academy of Science, Engineering and Technology, Vol 7, 2013.

14. Sharma A.K. \& Mittal N.D., Review on stress and vibration analysis of composite plates. Journal of Applied Sciences, 10 (23), 3156-3166, 2010.

15. Sharma A.K. \& Mittal N.D., Sharma A., Free vibration analysis of moderately thick antisymmetric cross-ply laminated rectangular plates with elastic edge constraints. International Journal of Mechanical Sciences, 53, 688 695, 2011.

16. Sharma A. K. \& Mittal N. D., Free vibration analysis of laminated composite plates with elastically restrained edges using FEM. Central European Journal of Engineering. 3 (2), 306-315, 2013.

17. Zhao X., Lee Y.Y., Liew K.M., Free vibration analysis of functionally graded plates using the element-free kp-Ritz method, Journal of Sound and Vibration 319 918-939 (2009). 